\title{
High Density Lipoprotein Cholesterol Measurement
}

National Cancer Institute

\section{Source}

National Cancer Institute. High Density Lipoprotein Cholesterol Measurement. NCI

Thesaurus. Code C105587.

The determination of the amount of high-density lipoprotein cholesterol present in a sample. 